\title{
A novel human TINP1 gene promotes cell proliferation through inhibition of p53 and p21 expression
}

\author{
WEI LI ${ }^{1}$, AI-PING SONG ${ }^{2}$, FANG ZHAO ${ }^{3}$, YONG-MEI HU ${ }^{1}$ and MU HUA ${ }^{4}$ \\ ${ }^{1}$ Biomedical Engineering Institute, School of Control Science and Engineering, Shandong University, \\ Jinan, Shandong 250061; ${ }^{2}$ Department of Ophthalmology, Second People's Hospital of Jinan, \\ Shandong 250021, P.R. China; ${ }^{3}$ Department of Medicine, Weill Medical College of Cornell University, New York, \\ NY 10021, USA; ${ }^{4}$ Shandong Institute of Scientific and Technical Information, Jinan, Shandong, P.R. China
}

Received April 20, 2013; Accepted June 25, 2013

DOI: $10.3892 / o r .2013 .2647$

\begin{abstract}
Transforming growth factor (TGF)- $\beta$-inducible nuclear protein 1 (TINP1) is a novel gene, which is localized at chromosome 5q13 where frequent abnormalities in hairy cell leukemia (HCL) occur. The present study investigated the effects of TINP1 knockdown or overexpression on the viability and gene expression of various tumor cell lines. siTINP1 was designed to knock down TINP1 expression. Reverse transcription polymerase chain reaction (RT-PCR) and western blotting were performed to assess gene expression; the cell counting kit-8 (CCK-8) assay was used to detect cell viability, and luciferase and flow cytometry assays were used to determine gene activity. TINP1 was widely expressed in various cell lines. In addition, TINP1 siRNA was able to knock down TINP1 expression in HeLa cells. TINP1 overexpression significantly promoted tumor cell proliferation, which may be associated with the downregulation of p53 expression. Furthermore, TINP1 promoted a number of cell lines to the $\mathrm{S}$ phase of the cell cycle. TINPI promotes cell proliferation and significantly reduces $\mathrm{p} 53$ and $\mathrm{p} 21$ expression.
\end{abstract}

\section{Introduction}

The novel proliferation-related gene transforming growth factor (TGF)- $\beta$-inducible nuclear protein 1 (TINP1) was recently identified and cloned by using the gene-function screening platform of the fluorescein reporter gene-phenotype system (1). TINP1 (GenBank accession number: NM_014886) is localized at chromosome $5 q 13$, which shows frequent abnormalities in hairy cell leukemia (HCL), indicating that this chromosomal region harbors a gene involved in the transformational events of HCL (2). Indeed, the cell viability and the cell cycle analysis have shown that TINP1 promotes HeLa cell

Correspondence to: Dr Wei Li, Biomedical Engineering Institute, School of Control Science and Engineering, Shandong University, Jinan, Shandong 250061, P.R. China

E-mail: cindy@sdu.edu.cn

Key words: TINP1, cell proliferation, p53 proliferation, which provides a basis for the further study of this gene's function. The TINPI gene covers 1,105 bp with 6 exons and 5 introns for coding a protein containing 260 amino acids $(1,3,4)$. The PSORT II program predicted that TINPI may be a nuclear protein $(91.3 \%)$ and that the TINPl gene contains a Ribosomal_S8e conserved domain at amino acids 1-237 after searching the Pfam database. Ribosomal_S8e is a very highly conserved domain in eukaryotes, and its function is involved in RNA-protein interactions that are associated with mRNA translation and protein synthesis $(3,5)$. In addition, a previous study indicated that the human TINPI gene is homologous to the NSA2 gene in Saccharomyces cerevisiae (6). The NSA2 gene has been demonstrated to play an important role in Saccharomyces cerevisiae growth, and it has been shown that the TINP1 gene exhibits similar functions to those of NSA2, which significantly promotes the growth of gene-deficient Saccharomyces cerevisiae (2). However, the role of TINP1 in human carcinogenesis and cancer progression remains largely unknown. It is clear that cell proliferation is an essential characteristics of tumor initiation and progression. Cell proliferation is due to cell cycle progression, which is specifically regulated in a time- and space-dependent manner, and abnormal cell proliferation induces the development of multiple diseases $(4,7,8)$, including human cancer. Thus, the present study investigated the signaling transduction pathway involved in human TINPI gene promotion of cell proliferation and the association between TINP1 expression and multiple proliferation-related signaling pathways. Furthermore, the molecular mechanisms responsible for the TINP1-mediated gene pathway were explored to provide a potential drug target for cancer treatment.

\section{Materials and methods}

Cell lines and culture. A cervical cancer HeLa cell line, an osteosarcoma U2OS cell line, a colorectal cancer HCT-16 cell line, a lung cancer H1299 cell line, and a hepatocellular carcinoma HepG2 cell line were maintained in our laboratory. A lung cancer A549 cell line, a human embryonic kidney HEK293T cell line, a Jurkat cell line and a THP-1 cell line were purchased from the Institute of Biochemistry and Cell Biology, Shanghai Institutes of Biological Sciences, Chinese Academy of Sciences (Shanghai, China). These cell lines were 
cultured in Dulbecco's modified Eagle's medium (DMEM) with $10 \%$ fetal bovine serum (FBS) at $37^{\circ} \mathrm{C}$ in an incubator with $5 \% \mathrm{CO}_{2}$ and $95 \%$ air.

Design of siTINP1 and gene transfection. TINP1 small interfering RNA (si-TINP1) and non-silencing control siRNA were designed and purchased from Santa Cruz Biotechnology, Inc. (Santa Cruz, CA, USA). The non-silencing siRNA was confirmed to have no matches with the complete human genome by a BLAST search in NCBI (www.nibi.nlm.nih.gov). These siRNAs were used to transfect tumor cells, i.e., tumor cells were seeded into 6-well plates at a concentration of $2.5 \times 10^{5}$ cells $/ \mathrm{ml}$ and grown in DMEM containing $10 \%$ FBS for $24 \mathrm{~h}$. siTINP1 and TINP1 cDNA containing plasmid or non-silencing siRNA (negative control) were transfected with Lipofectamine 2000 transfection reagent (Invitrogen, Carlsbad, CA, USA) according to the manufacturer's instructions.

Reverse transcription-polymerase chain reaction (RT-PCR). Total cellular RNA from cultured tumor cells was isolated using the TRIzol ${ }^{\mathrm{TM}}$ reagent (Invitrogen), according to the manufacturer's instructions, and reversely transcribed into single-strand cDNA using the Reverse transcript ${ }^{\mathrm{TM}}$ kit (Invitrogen). Next, PCR amplification was performed using specific primers for the TINPI gene: 5'-CTG CTG GAC AGA GAG GGA CAA-3' and 5'-TTT CTC CCT GGG CAC GTA CTT-3'. The housekeeping gene GAPDH was used as a loading control of PCR amplification using the following primers: 5'-TGA AGG TCG GAG TCA ACG GAT TTG GT-3' and 5'-CAT GTG GGC CAT GAG GTC CAC CAC-3'. The RT-PCR amplification was performed with the ThermoScript RT-PCR system (Invitrogen), according to the manufacturer's protocol, with an initial cycle of $94^{\circ} \mathrm{C}$ for $5 \mathrm{~min}$ and 35 cycles of $94^{\circ} \mathrm{C}$ for $30 \mathrm{sec}, 58^{\circ} \mathrm{C}$ for $30 \mathrm{sec}$, and $72^{\circ} \mathrm{C}$ for $30 \mathrm{sec}$, and a final extension at $72^{\circ} \mathrm{C}$ for $10 \mathrm{~min}$. The PCR products were separated in $2 \%$ agarose gel and semi-quantified according to the levels of GAPDH mRNA. Then, the TINP1 expression was compared with that of the negative control siRNA or vector only-transfected cells.

Protein extraction and western blot analysis. Total cellular protein was extracted from the cultured cells using a RIPA lysis buffer $(50 \mathrm{mM}$ Tris-HCl, pH 7.4; $150 \mathrm{mM} \mathrm{NaCl} ; 1 \%$ deoxycholate Na; $1 \% \mathrm{NP}-40 ; 0.1 \%$ sodium dodecyl sulfate, with freshly added protease inhibitor cocktail). Protein concentrations were determined using the BCA protein assay reagent (Pierce, Biotechnology, Inc., Rockford, IL, USA). Following quantification, $20 \mu \mathrm{g}$ of total protein samples were separated using $12 \%$ sodium dodecyl sulfate-polyacrylamide gel electrophoresis (SDS-PAGE) and transferred onto a nitrocellulose membrane (Hybond $^{\mathrm{TM}}$, ECLTM; Amersham Pharmacia, Little Chalfont, UK). After blocking for $2 \mathrm{~h}$ in $5 \%$ non-fat milk in Tris-buffer supplemented with $0.05 \%$ Tween-20 (TBS-T), the membrane was incubated with the corresponding primary antibody at $4^{\circ} \mathrm{C}$ overnight. Polyclonal antibodies against p53, Erk 1/2, JNK/ SPAK, p38, and $\beta$-actin were obtained from Cell Signaling Technology (Boston, MA, USA). The membrane was then rinsed in TBS-T three times for $10 \mathrm{~min}$ each and incubated with the secondary antibody at room temperature for $2 \mathrm{~h}$. The membrane was rinsed again in TBS-T three times for $10 \mathrm{~min}$
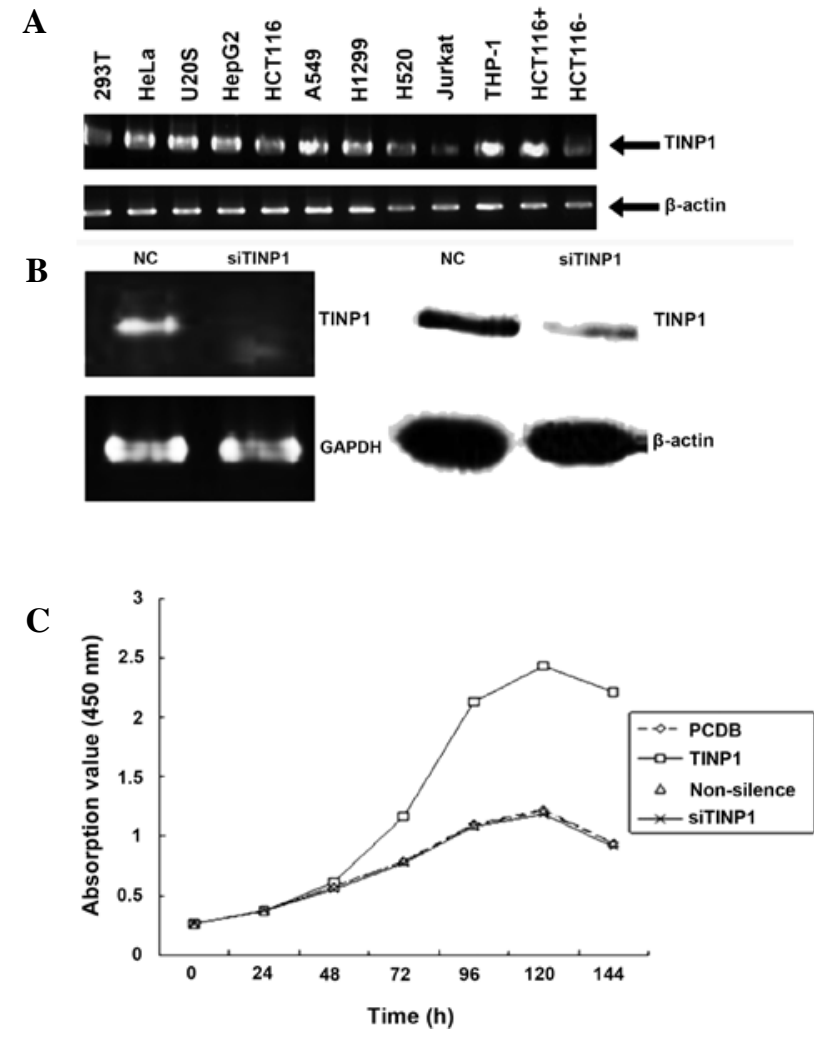

Figure 1. Knockdown of TINP1 expression in HeLa cells. (A) Expression of TINP1 mRNA in multiple cell lines; (B) knockdown of TINP1 mRNA and protein expressions in HeLa cells using siTINP1 nucleotides; (C) knockdown of TINP1 expression using siTINP1 reduced HeLa cell viability.

each and visualized using an ECL chemiluminescence system (Bio-Rad Laboratories, Hercules, CA, USA).

Cell viability cell counting kit-8(CCK-8) assay. The cells were seeded into 96 -well plates at a density of $2 \times 10^{3}$ cells $/ \mathrm{ml}$ in a volume of $100 \mu \mathrm{l} /$ well and placed in an incubator for 18-24 h. After reaching $40-60 \%$ confluency, the cells were transfected with $20 \mathrm{ng}$ of blank vector as a negative control, TINP1 plasmid, and H-Ras (as a positive control), respectively, using the VigoFect transfection reagent in triplicate and then cultured for 5 days. At the end of the experiments, $10 \mu \mathrm{l}$ of CCK- 8 reagent was added to each well, and the cells were further incubated for $2 \mathrm{~h}$. Subsequently, the absorbance value was determined using a multifunctional plate reader (GENios Pro $\left.{ }^{\mathrm{TM}}\right)$. Cell viability was summarized as the percentage of control.

Flow cytometry. The cells were seeded into 6-well plates at a density of $3 \times 10^{5}$ cells $/ \mathrm{ml}$ and transfected with different plasmids for up to 3 days. At the end of the experiments, the cells were digested with $2.5 \%$ pancreatin for $24 \mathrm{~h}$, harvested and prepared into single-cell suspensions. After washing with pre-cooled phosphate-buffered saline (PBS) twice, the cell suspension was rotated on a Vortex rotator; cooled $75 \%$ ethanol was added dropwise to the suspension and the cells were fixed at $-20^{\circ} \mathrm{C}$ overnight. The next day, the cell suspension was washed with PBS twice, incubated with RNase $(10 \mu \mathrm{g} / \mathrm{ml})$ at $37^{\circ} \mathrm{C}$ for $30 \mathrm{~min}$ and stained with propidium iodide. The cells were then analyzed by a FACSCalibur flow cytometer (Becton-Dickinson, Franklin Lakes, NJ, USA). 
A
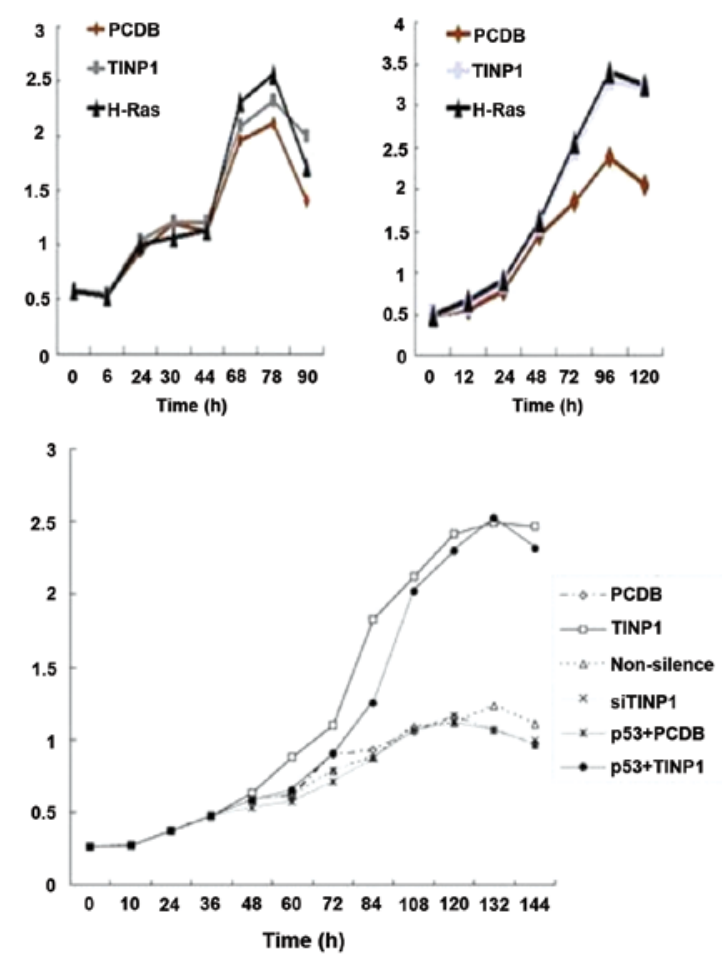

B

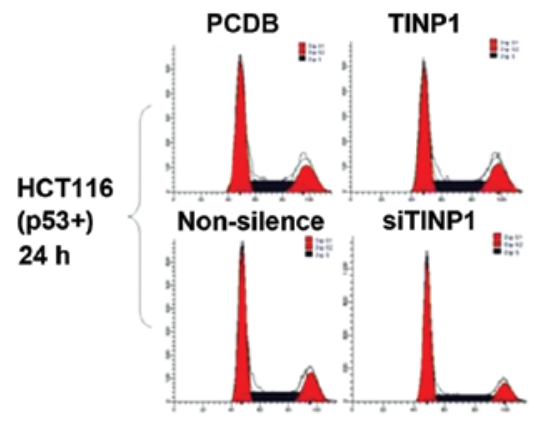

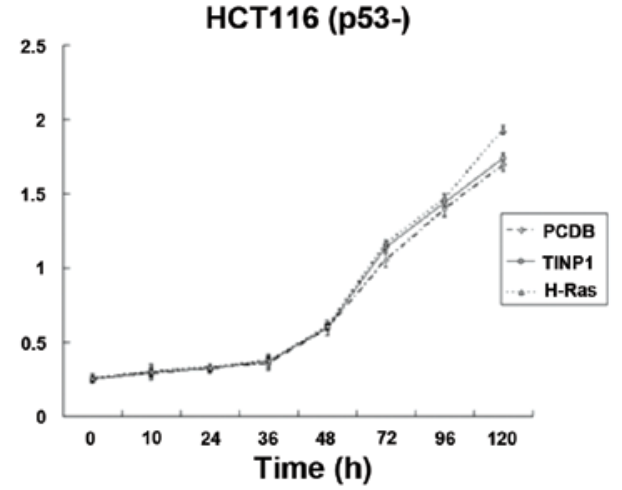
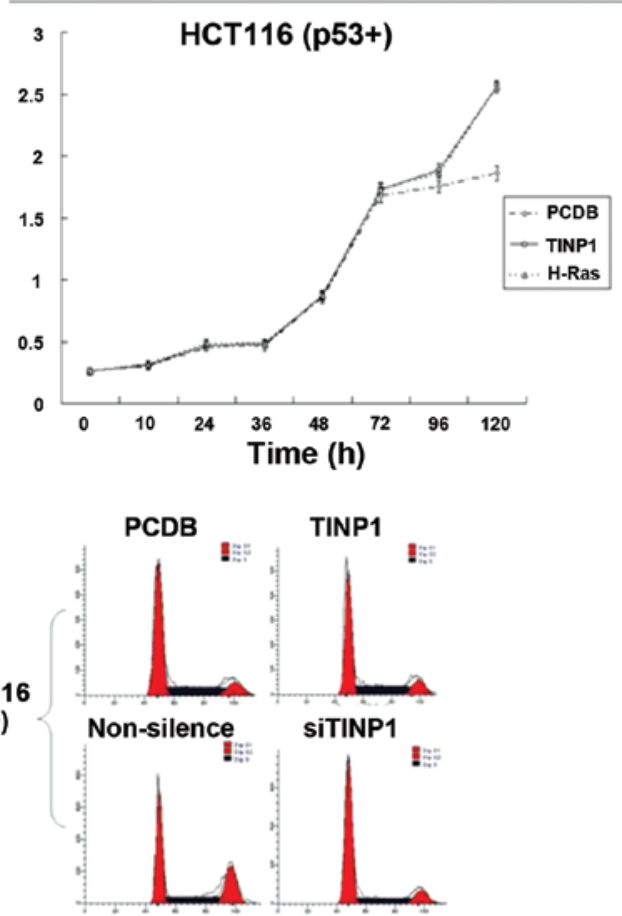

\begin{tabular}{|c|c|c|c|c|c|c|c|}
\hline \multirow{2}{*}{ Time } & Gene/siRNA & G1\% & S+G2\% & $\begin{array}{c}\text { Increased } \\
\text { S+G2\% }\end{array}$ & STD & n/experiment & P-value \\
\hline \multirow{5}{*}{ HCT116(p53+)-24 h h } & PCDB & 51.59 & 48.41 & - & - & 3 & - \\
\cline { 2 - 9 } & TINP1 & 47.69 & 52.31 & 3.9 & 0.71 & 3 & $<0.05$ \\
\cline { 2 - 9 } & Non-silence & 53.89 & 46.11 & - & - & 3 & - \\
\cline { 2 - 8 } & siTINP1 & 60.28 & 39.72 & -6.39 & 1.21 & 3 & $<0.05$ \\
\hline \multirow{5}{*}{ HCT116(p53+)-48 h h } & PCDB & 59.06 & 40.94 & - & - & 3 & - \\
\cline { 2 - 8 } & TINP1 & 54.15 & 45.85 & 4.91 & 0.86 & 3 & $<0.05$ \\
\cline { 2 - 8 } & Non-silence & 43.41 & 56.59 & - & - & 3 & - \\
\cline { 2 - 8 } & siTINP1 & 65.9 & 34.1 & -22.49 & 4.97 & 3 & $<0.05$ \\
\hline
\end{tabular}

Figure 2. Effect of TINP1 cDNA transfection in promoting tumor cell viability and cell cycle progression. (A) Cell viability assay; (B) flow cytometry assay.

Luciferase reporter assay. The cells were seeded into 96-well plates at a density of $1.1 \times 10^{6}$ cells $/ \mathrm{ml}$ in a volume of $100 \mu \mathrm{l} / \mathrm{well}$ and placed in an incubator for 18-24 h. After reaching 40-60\% confluency, the cells were co-transfected with the cell proliferation-related pathway reporters: $50 \mathrm{ng}$ of NF-kB-LUC, pWNT-LUC, pP53-LUC, pC/EBP-LUC, or pT-bet-LUC plus $50 \mathrm{ng}$ of pAP-1-LUC, $5 \mathrm{ng}$ of pRL-TK-LUC, and $50 \mathrm{ng}$ of TINP1 or the control plasmid using the VigoFect transfection reagent (Beijing Vigorous, Beijing, China). Cells transfected with blank vector served as blank controls, while cells transfected with TNF- $\alpha, \beta$-catenin, p53, PKA, aj41 and MEKK served as positive controls. These transfections were performed in triplicate and repeated at least once. Following incubation for $24 \mathrm{~h}$, the luciferase activity was determined using a multifunctional plate reader (GENios $\mathrm{Pro}^{\mathrm{TM}}$ ). The relative fluorescence value of the cells transfected with blank vector was defined as $100 \%$, and the results were expressed as the mean value of three replicate wells.

Statistical analyses. The data are summarized as means \pm standard deviation (SD) as appropriate. The Student's t-test was used to generate a P-value using SPSS software (SPSS, Inc., Chicago, IL, USA). A P-value $<0.05$ was considered to indicate a statistically significant difference. 

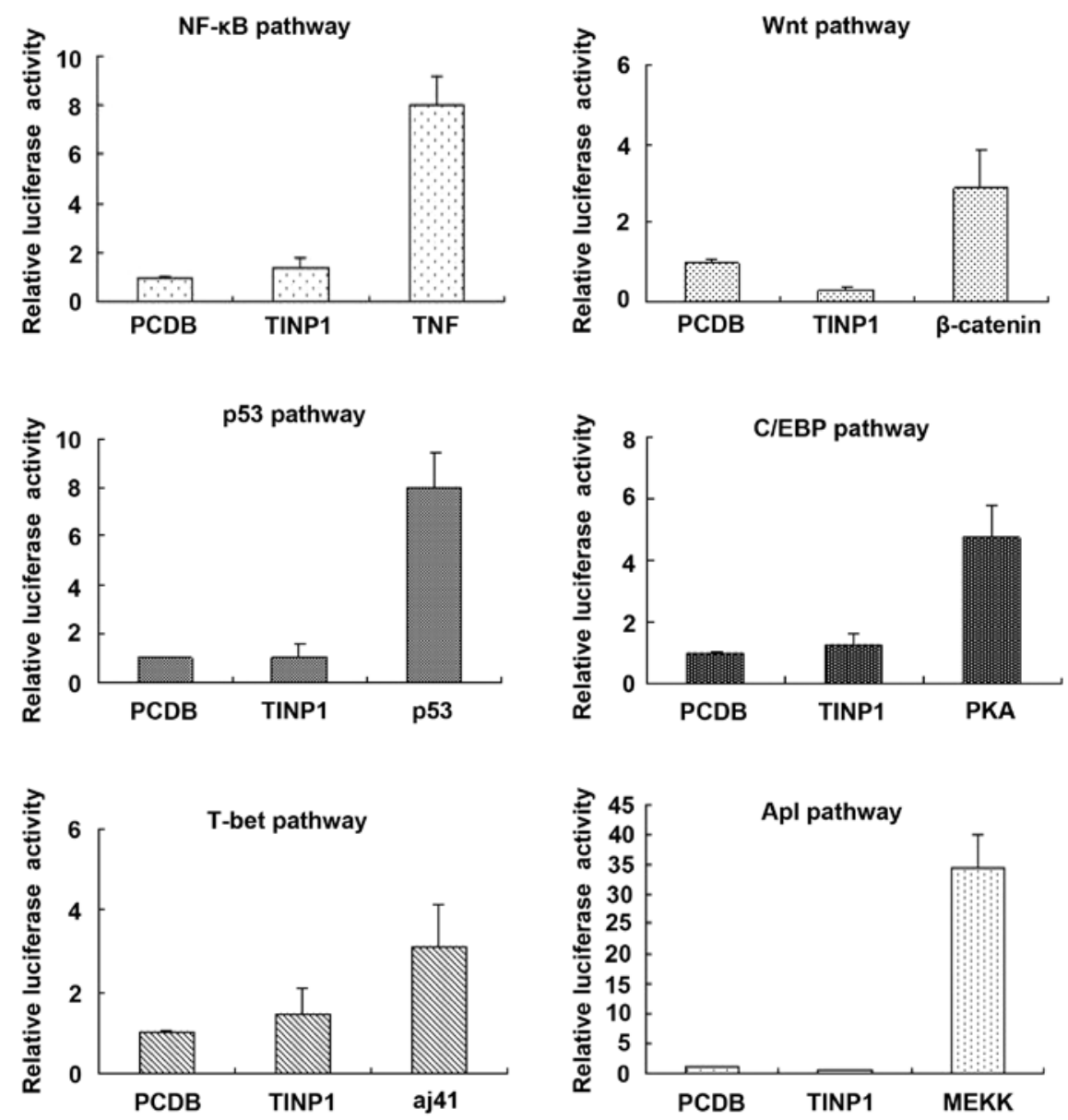

Figure 3. Effects of TINP1 overexpression on the regulation of different gene pathways.

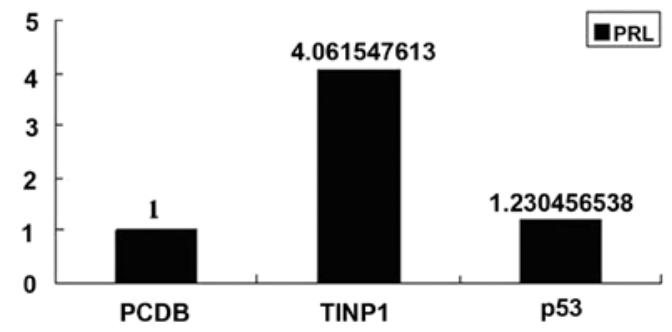

Figure 4. Effect of TINPI overexpression on the regulation of $p 53$ pathway activity.

\section{Results}

Expression of TINP1 and knockdown of TINP1 expression using siTINP1. In the present study, we first detected TINP1 expression in multiple cell lines and found that TINP1 was expressed in most of these cells using RT-PCR (Fig. 1A). Next, we chose HeLa cells for knockdown of TINP1 expression using TINP1 siRNA nucleotides. After 24 and $48 \mathrm{~h}$ of gene transfection, the endogenous TINP1 mRNA expression in siTINP1-transfected cells was significantly reduced compared to that in negative control cells (Fig. 1B). Next, we assessed the effects of TINP1 knockdown on the regulation of HeLa cell viability and found that siTINP1 significantly reduced tumor cell viability (Fig. 1C).
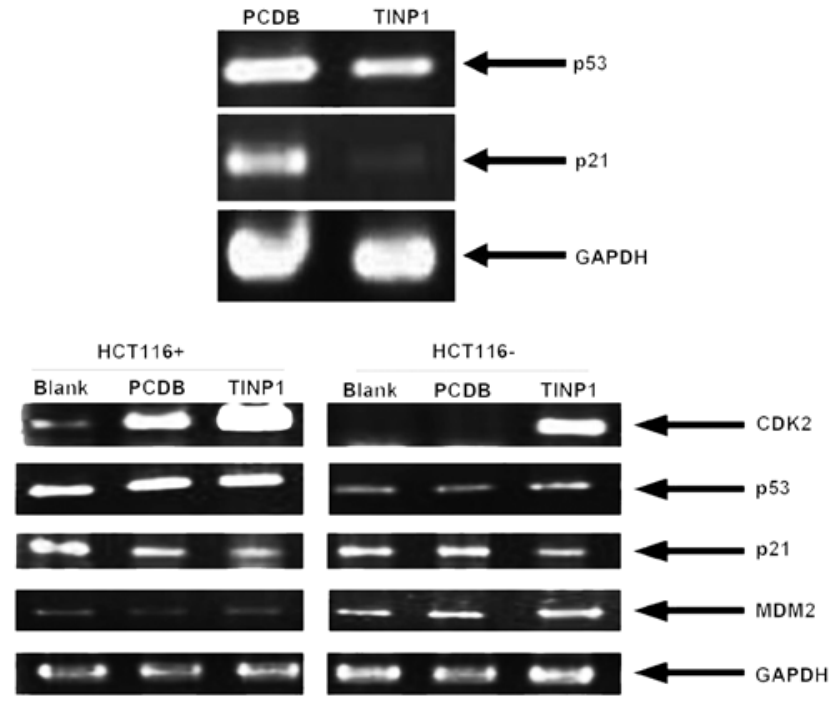

Figure 5. Effect of $T I N P 1$ on the regulation of $p 53$ and $p 21$ mRNA expression.

Effect of TINP1 overexpression on tumor cell viability and cell cycle progression. We also assessed TINP1 overexpression on the regulation of cell proliferation by transfecting TINP1 cDNA into HeLa cells and found that TINP1 promoted cell growth (Fig. 2A). In addition, flow cytometry data showed that TINP1 induced more cells at the $S$ phase of the cell cycle, further indicating cell proliferation (Fig. 2B). 


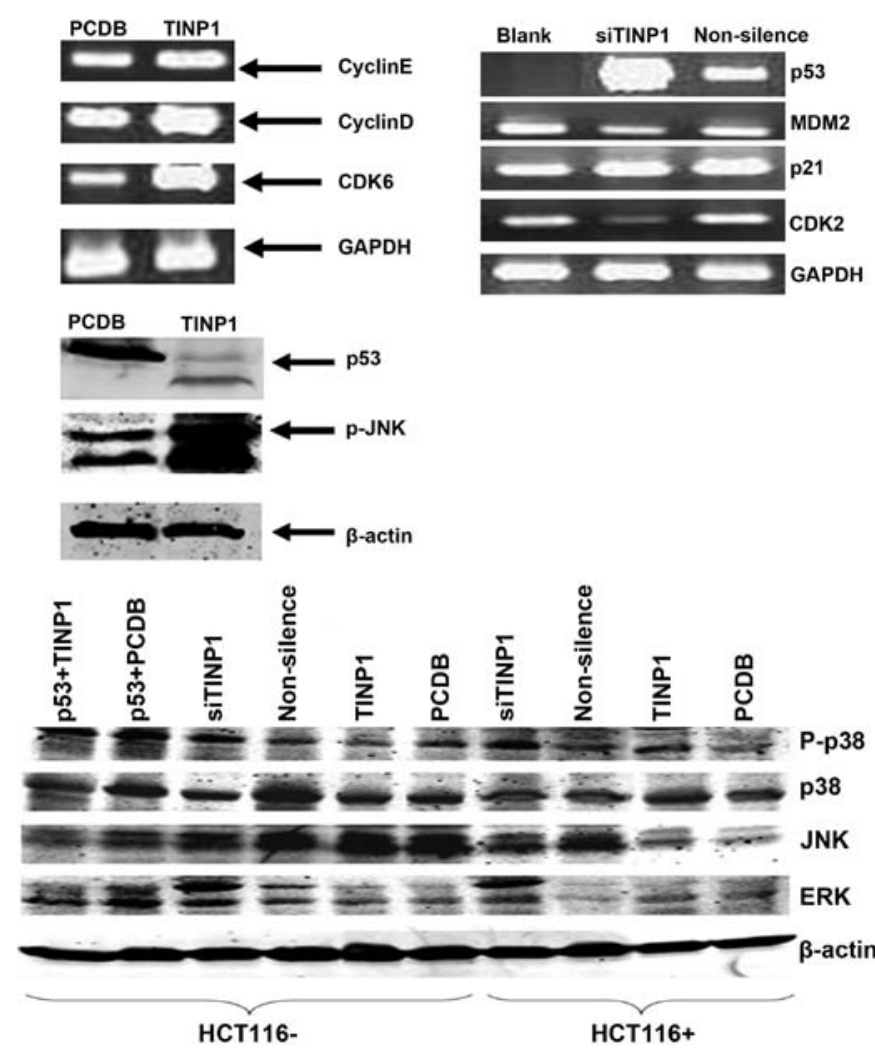

Figure 6. Effect of endogenous TINP1 expression on the regulation of p53 expression and CDK2 activation.

Effect of TINP1 overexpression on signaling pathways. To explore the role of TINP1 in signaling pathway regulation, we performed a dual-luciferase reporter assay to investigate six signaling pathways (including NF- $\mathrm{kB}$, WNT, p53, C/EBP, T-bet and AP1). The data showed that TINPI did not significantly alter the activities of these six signaling pathways (Fig. 3). However, TINP1 significantly promoted pRL-TK-LUC activity in the p53 pathway (Fig. 4). Next, we determined the expression of these p53-related genes and found that TINP1 significantly inhibited p53 mRNA levels, silenced p 21 mRNA expression in HCT-116 cells, and induced cyclin D1 and CDK6 expression (Figs. 5 and 6). By contrast, TINP1 siRNA induced p53 and p21 expression but reduced the expression of MDM2 and CDK2 mRNA (Fig. 5).

\section{Discussion}

In the present study, we investigated the effects of TINP1 knockdown and overexpression in the regulation of cell growth and gene expression in various cell lines. We found that TINP1 was expressed in multiple cell lines and that knockdown of TINP1 expression reduced HeLa cell proliferation. By contrast, the overexpression of TINP1 promoted cell proliferation in various cell lines, which was associated with the inhibition of $\mathrm{p} 21$ and $\mathrm{p} 53$ expression to promote the $\mathrm{S}$ phase of the cell cycle. However, TINP1 overexpression did not affect the activities of five different signaling pathways (NF- $\kappa \mathrm{B}, \mathrm{WNT}, \mathrm{C} / \mathrm{EBP}, \mathrm{AP} 1$ and T-bet). These data suggest that TINP1 plays a role in cell growth by regulating the expression of p53 and p21-related genes. Future studies will further investigate how TINP1 affects p53 and p21 gene expression so that targeting TINP1 expression in tumor cells may be used in clinical settings as a novel strategy to treat cancer.

The data from the present study clearly show that TINP1 overexpression significantly promoted cell proliferation and advanced the cells to the $\mathrm{S}$ phase of the cell cycle, whereas TINP1 knockdown reduced HeLa cell viability. These data confirmed a previous study that indicated a proliferative role of TINP1 in cells, since the human TINPI gene is a homolog of the NSA2 gene in Saccharomyces cerevisiae. The NSA2 gene plays a role in the growth of Saccharomyces cerevisiae (2). Furthermore, the SymAtlas online application revealed that the human TINPI gene is expressed in multiple tissues (2). Indeed, our present study demonstrated that TINPI was expressed in multiple cell lines. Since cell proliferation (i.e., growth) is involved in multiple gene signaling pathways, we assessed the role of TINPI in the activation of six different gene pathways. Our data showed that TINP1 does not alter the luciferase activities of NF- $\kappa \mathrm{B}, \mathrm{WNT}, \mathrm{C} / \mathrm{EBP}, \mathrm{AP} 1$ or T-bet genes. However, further study showed that the overexpression of TINP1 inhibited the expression of p53 and p21 mRNA but induced cyclin D1 and CDK6 expression, whereas knockdown of TINP1 induced p53 and p21 expression. Our present study linked TINP1 and the p53 gene pathway as promoting cell cycle progression. Further studies will investigate how TINP1 inhibits p53 and p21 expression and will also examine TINP1 as a novel target in cancer therapy.

\section{Acknowledgements}

This study was supported in part by grants from the National Natural Science Foundation (no. 30900730) and the Shandong Province Natural Science Foundation (Q2007D01).

\section{References}

1. Wu X, Ivanova G, Merup M, et al: Molecular analysis of the human chromosome 5 q13.3 region in patients with hairy cell leukemia and identification of tumor suppressor gene candidates. Genomics 60: 161-171, 1999.

2. Ohnishi Y, Saika S, Yamanaka O, et al: Investigation of mechanism of cell proliferation regulation and its clinical application. Nihon Ganka Gakkai Zasshi 109: 865-884, 2005 (In Japanese).

3. Stanchi F, Bertocco E, Toppo S, et al: Characterization of 16 novel human genes showing high similarity to yeast sequences. Yeast 18: 69-80, 2001.

4. Strausberg RL, Feingold EA, Grouse LH, et al: Generation and initial analysis of more than 15,000 full-length human and mouse cDNA sequences. Proc Natl Acad Sci USA 99: 16899-16903, 2002.

5. Saveanu C, Namane A, Gleizes PE, et al: Sequential protein association with nascent $60 \mathrm{~S}$ ribosomal particles. Mol Cell Biol 23: 4449-4460, 2003.

6. Zhang H, Ma X, Shi T, Song Q, Zhao H and Ma D: NSA2, a novel nucleolus protein regulates cell proliferation and cell cycle. Biochem Biophys Res Commun 391: 651-658, 2010.

7. Huang SS and Huang JS: TGF- $\beta$ control of cell proliferation. J Cell Biochem 96: 447-462, 2005.

8. Chittaranjan S, McConechy M, Hou YC, Freeman JD, Devorkin L and Gorski SM: Steroid hormone control of cell death and cell survival: molecular insights using RNAi. PLoS Genet 5: e1000379, 2009. 